\title{
BMJ Open British OsteoNEcrosis Study (BONES) protocol: a prospective cohort study to examine the natural history of osteonecrosis in older children, teenagers and young adults with acute lymphoblastic leukaemia and lymphoblastic lymphoma
}

\author{
Nadia Amin, ${ }^{\oplus 1}$ Sally Kinsey, ${ }^{1,2}$ Richard Feltbower, ${ }^{3}$ Jeannette Kraft, ${ }^{4}$ \\ Elizabeth Whitehead, ${ }^{5}$ Mark Velangi, ${ }^{6}$ Beki James ${ }^{2}$
}

To cite: Amin N, Kinsey S, Feltbower R, et al. British OsteoNEcrosis Study (BONES) protocol: a prospective cohort study to examine the natural history of osteonecrosis in older children, teenagers and young adults with acute lymphoblastic leukaemia and lymphoblastic lymphoma. BMJ Open 2019;9:e027204. doi:10.1136/ bmjopen-2018-027204

- Prepublication history and additional material for this paper are available online. To view these files, please visit the journal online (http://dx.doi org/10.1136/bmjopen-2018027204).

Received 17 0ctober 2018 Revised 25 February 2019 Accepted 10 April 2019

Check for updates

(c) Author(s) (or their employer(s)) 2019. Re-use permitted under CC BY-NC. No commercial re-use. See rights and permissions. Published by BMJ.

For numbered affiliations see end of article.

Correspondence to

Dr Nadia Amin;

nadia@cantab.net

\section{ABSTRACT}

Introduction 0steonecrosis is a well-recognised treatment-related morbidity risk in patients diagnosed with acute lymphoblastic leukaemia (ALL) and lymphoblastic lymphoma (LBL), with a high rate of affected patients requiring surgical intervention. Patients may have asymptomatic changes on imaging studies that spontaneously regress, and little is known about the natural history of osteonecrotic changes seen. The main aim of the British 0steoNEcrosis Study (BONES) is to determine the incidence of symptomatic and asymptomatic osteonecrosis in the lower extremities of survivors of ALL or LBL diagnosed aged 10-24 years in the UK at different time points in their treatment. This study also aims to identify risk factors for progression and the development of symptomatic osteonecrosis in this population, as well as specific radiological features that predict for progression or regression in those with asymptomatic osteonecrosis

Methods and analysis BONES is a prospective, longitudinal cohort study based at principal treatment centres around the UK. Participants are patients aged 10-24 years diagnosed with ALL or LBL under standard criteria. Assessment for osteonecrosis will be within 4 weeks of diagnosis, at the end of delayed intensification and 1, 2 and 3 years after the start of maintenance therapy. Assessment will consist of MRI scans of the lower limbs and physiotherapy assessment. Clinical and biochemical data will be collected at each of the time points. Bone mineral density data and vertebral fracture assessment using dual-energy $\mathrm{X}$-ray absorptiometry will be collected at diagnosis and annually for 3 years after diagnosis of malignancy.

Ethics and dissemination Ethical approval has been obtained through the Yorkshire and Humber Sheffield Research Ethics Committee (reference number: 16/ $\mathrm{YH} / 0206)$. Study results will be published on the study website, in peer-reviewed journals and presented at relevant conferences and via social media.
Strengths and limitations of this study

- This study will be the first UK prospective study to obtain MRI within 4 weeks of diagnosis of acute lymphoblastic leukaemia (ALL), with sequential imaging at four further time-points to assess progression or regression of osteonecrotic lesions.

- This study targets the most vulnerable patient population, those aged 10-24 years, who are at highest risk of development of symptomatic osteonecrosis.

- It will simultaneously assess multiple domains to correlate physical signs, symptoms and biological markers with MRI changes.

- This study is limited by the anticipated small sample size, which is due to the rarity of ALL and lymphoblastic lymphoma in patients over 10 years of age, and prospective imaging of lower extremities only.

Trial registration number NCT02598401; Pre-results.

\section{INTRODUCTION}

Survival from acute lymphoblastic leukaemia (ALL) and lymphoblastic lymphoma (LBL) has steadily increased over the last 40 years so that the expected cure rate is now greater than $90 \%$ in children and young people presenting with ALL. ${ }^{1}$ This progress shifts the entire treatment paradigm so that the goal moves beyond cure to returning the young person to a normal life. The biggest barrier to this is the burden of treatment associated toxicity, and attention internationally is now beginning to focus on this issue. Osteonecrosis (previously also referred to as avascular necrosis, ischaemic necrosis and aseptic 
necrosis) can be a devastating complication of treatment in older children and teenagers treated for ALL and can cause significant long-term morbidity. ${ }^{2}$ However, despite increasing concern about osteonecrosis, our understanding of it in the context of ALL or LBL is limited. Historically, information about osteonecrosis has not been well captured in previous studies of ALL, which partly reflects lack of good definitions and incomplete retrospective reporting.

Osteonecrosis occurs when there is bone ischaemia and infarction caused by temporary or permanent disruption to the blood supply and in ALL typically affects the femoral head, humeral head, knee, shoulder and ankles. ${ }^{2}$ It is mostly an iatrogenic complication that has been attributed to increased use of glucocorticoids in treatment of ALL. ${ }^{3}$ The role of other agents, such as high dose methotrexate ${ }^{4}$ and asparaginase, ${ }^{5}{ }^{6}$ is uncertain. It has been reported that asparaginase reduces dexamethasone clearance and could potentiate the osteonecrotic effect of glucocorticoids. ${ }^{6}{ }^{7}$ The cumulative dose of received glucocorticoids in patients with ALL has been shown to correlate with the risk of osteonecrosis, ${ }^{8}$ but there is no clear increase in osteonecrotic risk with the administration of either prednisolone or dexamethasone. ${ }^{8-11}$ Development of osteonecrosis appears to be multifactorial but is seen more commonly in patients as survival improves and high dose steroids have become embedded in treatment regimens.

Glucocorticoids predispose to the development of osteonecrosis in a number of ways, with proposed aetiologies including:

- Creation of a hypercoagulable state with endothelial cell apoptosis and development of microthrombi.

- Suppression of osteoblasts and apoptosis of osteocytes impairing the bone repair process.

- Stimulation of intramedullary lipocyte proliferation and hypertrophy resulting in increased intraosseous pressure.

These factors combine to compromise blood circulation to the bone leading to cell death in a self-perpetuating cycle. ${ }^{12}$

Interosseous fat emboli with intravascular coagulation and osteonecrosis has been described, ${ }^{13}$ with an overload of subchondral fat emboli, hypercoagulability, stasis and endothelial damage by free fatty acids hypothesised to cause end organ damage. Glucocorticoids-causing dyslipidaemia may promote the formation of fat emboli, although fat emboli are also found in healthy bones, which do not go on to develop osteonecrosis. The role of hypercoagulability is unclear. Some studies have shown procoagulant abnormalities in patients with osteonecrosis, ${ }^{14}$ but the common thrombophilias have not been identified as risk factors for osteonecrosis, highlighting the multifactorial nature of the condition.

In one of the largest studies with prospective MRI screening to assess both symptomatic and asymptomatic osteonecrosis, the cumulative incidence of osteonecrosis involving the epiphysis or metaphysis of at least one hip was $17.1 \% \pm 1.8 \%$ after early screening (1 year) and $21.7 \% \pm 1.9 \%$ after completion of therapy (4 years).$^{15}$ By the end of therapy, extensive femoral head osteonecrosis affecting $\geq 30 \%$ of the epiphysial surface had developed in $6.5 \% \pm 1.1 \%$ of all patients and $24 \% \pm 4.4 \%$ of those aged over 10 years. ${ }^{15}$ The first findings of the osteonecrosis in pediatric patients with acute lymphoblastic leukaemia and lymphoblastic lymphoma (OPAL) trial, where MRI screening was at a median of 12.5 days, found leukaemic infiltrate at diagnosis was not associated with osteonecrotic lesions ${ }^{16}$ but the point at which asymptomatic lesions develop remains unclear.

There are many more reports that rely on proactive reporting to the study centre, with no prospective screening for asymptomatic osteonecrosis, and as expected, these tend to give a far lower prevalence of osteonecrosis, ranging from $0.67 \%$ to $15 \% .{ }^{17-23}$

Age has consistently been identified as the most significant risk factor for development of symptomatic osteonecrosis, with the greatest incidence of osteonecrosis occurring in patients between 10 years and 20 years of age at diagnosis of ALL, ${ }^{22-27}$ a time of rapid skeletal growth. The pathogenesis that puts this group at highest risk of development of osteonecrosis is uncertain, although factors such as hormonal changes, skeletal maturation, osseous blood vessel supply, dexamethasone clearance and changes in concentrations of coagulation factors may all play a role. ${ }^{728}$

There is no clear consensus on risk differences with sex of the patient, with variation in study findings. ${ }^{10} 15171822$ 29-41 Inconsistent results have also been reported regarding the influence of increased body mass index (BMI) as a risk factor for development of osteonecrosis, ${ }^{1532344041}$ and it is possible that varying thresholds used for statistical analysis effect likelihood of BMI being found as a risk factor. One prospective study has reported a higher cumulative incidence of osteonecrosis in patients with higher increases in total cholesterol and triglycerides during therapy. ${ }^{42}$ White race was found to be a risk factor in a number of studies, ${ }^{2533} 35$ but again this was inconsistent. ${ }^{1524}$ Ethnicity as a risk factor is a difficult area to study due to a number of confounding factors, variation in terminology and differences in how ethnic groups are categorised.

Various genetic risk factors for the development of osteonecrosis have been identified. Genome-wide association studies indicate the glutamate receptor pathway to be of crucial importance, and single-nucleotide polymorphisms in adipogenesis pathways and in enhancers active in mesenchymal stem cells were also significantly associated with osteonecrosis development. ${ }^{35} 43$ Glucocorticoid receptor binding sites have also been implicated in development of osteonecrosis. ${ }^{44}$

It is recognised that a significant percentage of changes on imaging studies identified as osteonecrosis may regress, ${ }^{24}$ although the reasons for this are not understood. It is possible that some radiological changes interpreted as representing steroid-associated osteonecrosis 


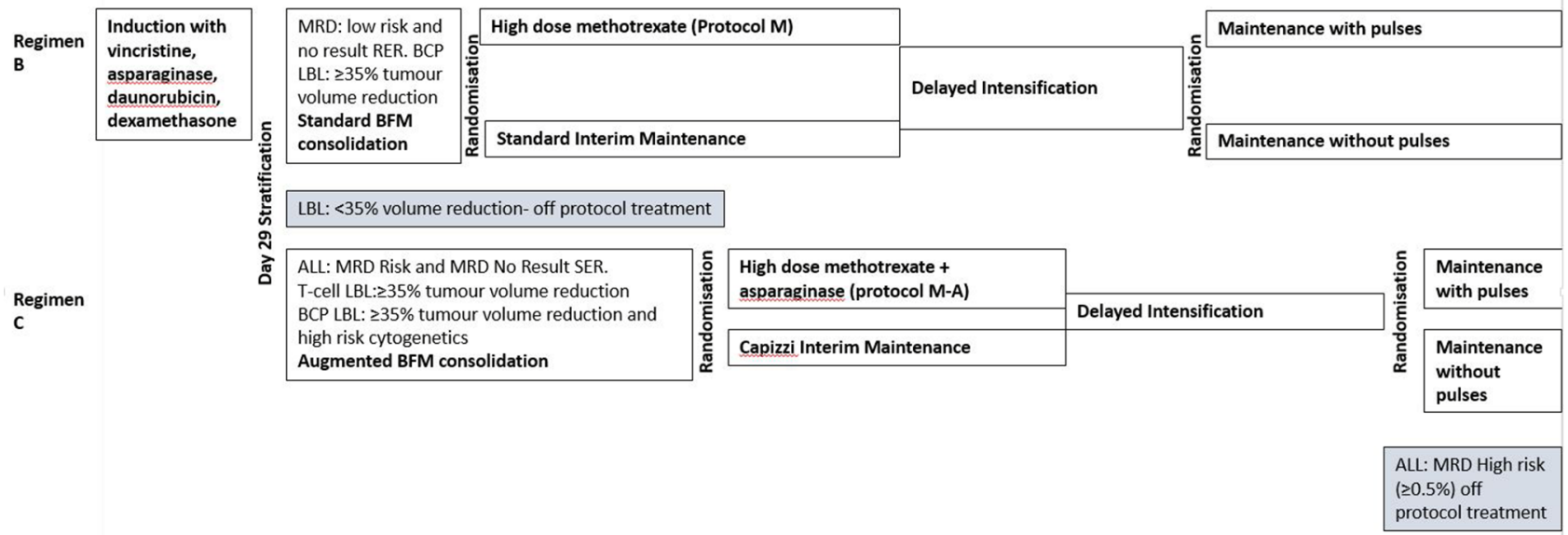

Figure 1 UKALL 2011 trial schema for patients over the age of 10 (excluding patients with Down's syndrome). BCP, B-cell precursor; BFM, Berlin-Frankfurt-Munich; LBL, lymphoblastic lymphoma; MRD, minimal residual disease; RER, rapid early response ( $<25 \%$ blasts at day 8 of induction); SER: slow early response ( $\geq 25 \%$ blasts at day 8 of induction).

are in fact changes that have been present at diagnosis and that are a consequence of the original leukaemia.

Currently, the most widely used radiological classification systems, such as the modified Ficat and Arlet, ${ }^{45}$ use a multimodal approach combining scores for X-ray, MRI and in some cases bone scan findings. Most widely used classification systems were developed specifically for changes in the femoral head, in some cases over 20 years ago and in an entirely different patient population. ${ }^{45-49}$ Further classifications systems have been developed more specifically for our patient population but as yet with no prognostic validation. ${ }^{50}$ This study will provide the data needed to develop and provide prognostic validation of a radiological classification system that correlates with clinical status, as well as provide greater understanding of the natural history of bone lesions in patients being treated for ALL or LBL. Only once this is done can meaningful intervention studies be initiated.

\section{Treatment for UK patients with ALL or LBL}

The majority of young people diagnosed with ALL or LBL between 26 April 2012 and 31 December 2018 consented to be part of the national trial, UKALL 2011 (ISRCTN64515327, Eudract 2010-020924-22), and treatment for patients aged between 10 years and 25 years at diagnosis of ALL or LBL is described in figure 1. A list of all chemotherapeutic agents are available in online supplementary file 1. Patients who did not consent to participate in UKALL2011, or who are diagnosed after the study closure, will receive the same treatment as those on the trial and at the point of randomisation receive standard interim or Capizzi interim maintenance, depending on their risk stratification. At the next randomisation point they receive maintenance therapy with vincristine/dexamethasone pulses and intrathecal methotrexate.
Postinduction treatment is determined by minimal residual disease (MRD) in ALL patients or tumour volume assessment in patients with LBL. Patients with no MRD results are assessed by morphology (\% of blasts at day 8 of induction).

If a patient has been randomised to high dose methotrexate therapy, they will have no subsequent intrathecal methotrexate in maintenance but can be randomised to either pulses or no pulses. An exception to this is that patients with $\mathrm{T}$ cell ALL with white cell count $>100 \times 10^{9}$ cells/L at diagnosis who have an additional six doses of intrathecal methotrexate in maintenance. Pulses consist of vincristine and dexamethasone. If they have been randomised to either standard or Capizzi interim maintenance, they will be randomised to maintenance therapy with or without pulses, and all patients will receive intrathecal methotrexate.

Treatment will last 2 years from the start of interim maintenance for female patients, and 3 years from the start of interim maintenance for male patients. There are some treatment modifications for patients with Down's syndrome to reduce toxicity.

\section{OBJECTIVES}

The objective is to establish a prospective, multicentre study for older children, teenagers and young adults that can address the following questions:

- What is the incidence of symptomatic and asymptomatic osteonecrosis in older children, teenagers and young adults being treated for ALL or LBL in the UK at different time points in their treatment?

- What are the risk factors for progression and the development of symptomatic osteonecrosis in this population? 
- Are there specific radiological features that predict for either progression or regression in those with asymptomatic osteonecrosis?

The study also aims to:

- Evaluate functional ability as measured by the Childhood Health Assessment Questionnaire (CHAQ) and physiotherapy assessment and explore the correlation of this with MRI findings to start to establish validity of use in patients with osteonecrosis.

- Evaluate changes in bone mineral density and vertebral fracture incidence during treatment for ALL or LBL.

\section{METHODS AND ANALYSIS}

Details of the protocol, data collection forms, consent forms and patient information leaflets are available at http://childhealth.leeds.ac.uk/bones.html.

\section{Study design}

Multicentre prospective longitudinal cohort study.

\section{Patient and public involvement}

Patients and families undergoing treatment or who had completed treatment for ALL or LBL were involved in the study design and in literature developed for patient information by use of semistructured interviews. Patients were not involved in the recruitment to and conduct of the study. Results will be disseminated to study participants via the British OsteNEcrosis Study (BONES) website.

\section{Study setting}

The BONES is conducted in principal treatment centres and teenage and young adult centres for patients with cancer within the UK. It is currently open in Leeds Children's Hospital; St James's Hospital, Leeds; Birmingham Children's Hospital; and Southampton Children's Hospital. Additional centres, including Children's Hospital for Wales, are in the research and development process.

\section{Dates of study}

The first site opened to recruitment on 10 April 2017. The most recent centre to join opened to recruitment on 22 March 2018. Additional sites are still in the process of opening the study. Recruitment is for a period of 2 years from site opening or until a total of 50 patients are recruited.

\section{Study population}

Inclusion criteria: children, teenagers or young adults between the age of 10 years and 24 years 364 days (at the time of diagnosis) with a first diagnosis of ALL or LBL (T-cell non Hodgkin lymphoma [TNHL] or Surface membrane immunoglobulin [SmIg] negative precursor B-cell non Hodgkin lymphoma [B-NHL]) diagnosed under standard criteria are eligible for BONES.

Exclusion criteria: inability to have MRI scans of lower limbs

\section{Recruitment target}

The recruitment target is 50 patients over a 2-year period, which is based on an anticipated participation of $75 \%$ of eligible cases. Given the observational nature of the study, and the wide number of potential predictors of interest, a power calculation is of limited relevance and is difficult to calculate given the current lack of data. However, taking pubertal status as an example, assuming $60 \%$ of patients will be in puberty, the study would detect a risk ratio of 3 with $82 \%$ power with a $5 \%$ level of significance.

\section{Study outcomes}

Primary outcome

- Cumulative incidence of symptomatic and asymptomatic osteonecrosis in patients aged between 10 and $<25$ years being treated for ALL or LBL in the UK at multiple time points in their treatment.

Key secondary outcomes

- Risk factors for progression and development of symptomatic osteonecrosis.

- Specific radiological features that predict for either progression or regression in those with osteonecrosis.

- Evaluation of functional ability as measured by CHAQ and physiotherapy assessment, with exploration of correlation with radiological findings.

- Bone mineral density changes as measured by dual-energy X-ray absorptiometry (DXA) during treatment for ALL or LBL.

- Prevalence and risk factors for development of vertebral fractures during treatment for ALL or LBL.

\section{Patient assessment}

Irrespective of symptoms patients will be screened for osteonecrosis via prospective MRI of the hips, knees and ankles at the following time points:

- Within 4 weeks of diagnosis.

- At the end of delayed intensification (typically 6-8 months after start of ALL treatment).

- One year after the start of maintenance.

- Two years after the start of maintenance.

- Three years after the start of maintenance.

Patients will also have a physiotherapy assessment at each of these time points, including subjective and objective assessments, with collection of clinical and biochemical data.

Where facilities exist, DXA scans and vertebral fracture assessment will be performed at diagnosis and annually for 3 years after diagnosis.

\section{Magnetic resonance imaging}

MRI of the lower limbs including hips, knees and ankles comprises unenhanced coronal T1-weighted and short tau inversion recovery images of $5 \mathrm{~mm}$ (or less) slice thickness as a minimum protocol. Scanning parameters may vary slightly depending on available MR scanners in each participating centre.

It can be difficult to differentiate osteonecrosis from other abnormalities affecting the bone such as marrow 
oedema, punctate foci of altered signal, haematopoietic marrow changes in children and, as we are imaging children with ALL, early leukaemic marrow infiltration. ${ }^{51}$ Osteonecrosis is defined as an area of yellow marrow surrounded by a low signal intensity rim on all pulse sequences or a double line rim comprising a low signal line and an adjacent high signal line on fluid sensitive sequences. The area of osteonecrosis may be complex in shape with serpentine, crescentic, band-like or undulating outline or represented as multiple small lesions. ${ }^{52-54}$ The presence of non-classical abnormalities will also be recorded if encountered, including haemorrhagic or cystic change as well as non-specific marrow changes and marrow oedema as these have been previously described and may represent significant prognostic factors. ${ }^{52-54}$

\section{Clinical and demographic data collection}

Baseline demographic data collection includes the child's age, sex, ethnic background (white British; Asian; black; mixed; other), postcode, height and weight at diagnosis. Clinical data are provided by the treating clinicians via a dedicated clinical report form, which includes information on pubertal status, highest white cell count prior to treatment, immunophenotype, cytogenetics and molecular results, along with presence or absence of hepatomegaly, splenomegaly, lymphadenopathy and bone pain at diagnosis.

At each of the time points outlined above, details regarding treatment regime, height, weight, phase of puberty and diagnosis and management of symptomatic osteonecrosis are collected. Data on results of routine blood tests, including lipid profile, albumin, bone profile, parathyroid hormone (PTH) and vitamin D levels is also collected. Clinicians collecting these details are blinded to the study MRI reports.

If a patient develops symptomatic osteonecrosis of upper or lower limbs, they will be managed as per local policy, but imaging results and clinical data will be collated.

\section{Physiotherapy evaluation}

The physiotherapy assessment consists of a paper questionnaire for completion by the participant, which includes information about activity levels, mobility, pain and the CHAQ, alongside a physical assessment evaluating gait, range of movement and muscle power. ${ }^{55}$ The CHAQ assesses three outcome dimensions: disability, discomfort and pain and is completed by self-report, requiring approximately $10-15 \mathrm{~min}$ to complete. It is most commonly used to assess health status and physical function in children with juvenile arthritis, for whom it is validated, ${ }^{55}$ but is also validated for use in children with chronic musculoskeletal pain, ${ }^{56}$ dermatomyositis ${ }^{57}$ and systemic lupus erythematosus. ${ }^{58}$

\section{Bone mineral density and vertebral fracture assessment}

Patients will undergo DXA scans with vertebral fracture assessment with collection of the following measurements: posterior-anterior lumbar spine (L1-4) and total body less head (TBLH), bone mineral apparent density (BMAD) and thoracic and lumbar vertebral fracture incidence.

A schema with BONES study procedures is presented in figure 2.

\section{Data analysis plan}

The report of this study will be prepared in accordance to guidelines set by the Strengthening the Reporting of Observational Studies in Epidemiology (STROBE) statement for observational studies. ${ }^{59}$

A central review panel consisting of paediatric radiologists with an interest in paediatric musculoskeletal imaging will review each MRI. The grade of osteonecrosis will be assessed using a modified scoring system by reference using a study radiology pro forma.

We will be using the classification system published by Niinimäki et $a \tilde{l}^{\tilde{0}}$ to assess osteonecrosis in the lower legs. As this system is not joint specific, it can be used to assess hips, knees and ankles in the same way. Our study radiology proforma will also separately record osteonecrosis seen within the metaphysis and diaphysis of long bones. If different scores are seen for two bones comprising a joint (eg, tibial and femoral epiphysis as part of the knee), both scores will be captured before giving the overall score for the knee according to Niinimäki et al, with the aim to assess the overall burden of osteonecrosis in the limb.

DXA and vertebral fracture assessment results will also be reviewed centrally, with adjustments to bone mineral density using bone mineral adjusted density bone mineral apparent density (BMAD) for the spine, and the height Z-score for TBLH. ${ }^{60}$ The thoracic and lumbar vertebra will be assessed (T4-L4 where possible), using the Genant semiquantitative method. ${ }^{61}$

The information from the CHAQ will be numerically coded using the disability index, global evaluation and pain assessment. The physiotherapy assessment will also be numerically coded to score muscle power and range of movement for each individual joint. Qualitative statements will be recorded and coded at the end of the study.

Data will be collected and analysed in clinically relevant categories, while $\chi^{2}$ tests and multivariable logistic regression models will be used to determine differences between groups adjusting for a relevant set of confounders identified using causal inference methods. ${ }^{62}$ Potential confounders that will be assessed include age, sex, ethnic group, socioeconomic status (using the Index of Multiple Deprivation $\operatorname{rank}^{63}$ ), treatment arm, highest white cell count, immunophenotype, cytogenetics (categorised into risk groups as per the UKALL 2011 protocol), phase of puberty, BMI Z-score, lipids, albumin, presence of vertebral fractures, bone mineral density, bone alkaline phosphatase (ALP), PTH and vitamin D status. ORs will be used to describe size of observed associations with $95 \%$ CIs. If numbers are sufficiently robust, a more sophisticated ordered logistic regression analysis will be carried out using an ordered categorical outcome variable for 


\begin{tabular}{|c|c|c|c|c|c|c|c|c|c|c|}
\hline & 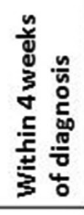 & 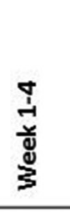 & 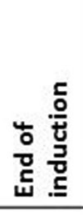 & 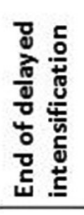 & 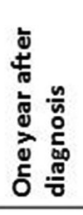 & 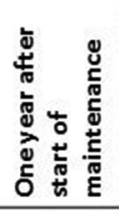 & 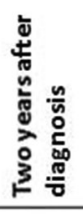 & 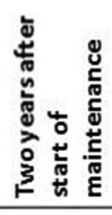 & 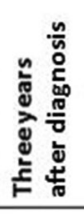 & 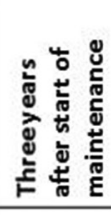 \\
\hline Consent & $\Delta$ & & & & & & & & & \\
\hline $\begin{array}{l}\text { MRI scan lower } \\
\text { limbs }\end{array}$ & & $\Delta$ & & $\Delta$ & & $\Delta$ & & $\Delta$ & & $\Delta$ \\
\hline $\begin{array}{l}\text { Routine blood } \\
\text { tests (LFTs, } \\
\text { calcium, } \\
\text { phosphate, } \\
\text { cholesterol, } \\
\text { triglycerides, } \\
\text { HDL, LDL } \\
\text { Vitamin D, PTH }\end{array}$ & & $\Delta$ & $\Delta$ & $\Delta$ & & 0 & & $\Delta$ & & $\Delta$ \\
\hline $\begin{array}{l}\text { Physiotherapy } \\
\text { assessment } \\
\text { including c- } \\
\text { HAQ }\end{array}$ & & $\Delta$ & & $\Delta$ & & 0 & & $\Delta$ & & $\Delta$ \\
\hline $\begin{array}{l}\text { DXA scan with } \\
\text { vertebral } \\
\text { fracture } \\
\text { assessment }\end{array}$ & & $\Delta$ & & & $\diamond$ & & $\diamond$ & & $\Delta$ & \\
\hline $\begin{array}{l}\text { Clinician } \\
\text { assessment }\end{array}$ & & $\Delta$ & & $\Delta$ & & $\Delta$ & & $\Delta$ & & $\Delta$ \\
\hline $\begin{array}{l}\text { End of } \\
\text { induction form }\end{array}$ & & & $\Delta$ & & & & & & & \\
\hline
\end{tabular}

Figure 2 Schema of BONES study procedures. BONES, British OsteoNEcrosis Study; CHAQ, Childhood Health Assessment Questionnaire; DXA, dual-energy X-ray absorptiometry; LFTs, liver function tests; LDL, low-density lipoprotein; HDL, highdensity lipoprotein.

severity of osteonecrosis, and risk of developing osteonecrosis will be assessed using Poisson regression, using the same set of confounders and the risk estimates, quantified by incidence rate ratios and $95 \%$ CIs.

Data completeness and validity

We will carry out range checks on the variables listed:

- Albumin.

- HDL.

- LDL.

- Cholesterol.

- Triglycerides.

- PTH.

- Vitamin D.

- ALP.

- Calcium.

- Phosphate.

If data on some subjects are missing at some time points, the entire subject history will not simply be excluded from analysis. The main patient characteristics will be described in terms of variable completeness by summarising the proportion of missing values. If numbers allow, levels of missingness will also be examined according to each recruiting centre. If the data are missing at rates higher than the expected attrition rate, the following steps will be taken:

- If data regarding independent variables are missing but data for the corresponding dependent variables are present, we will do multiple imputations for the missing values.

- If some data associated with a dependent variable are missing, such as some follow-up data, and the underlying mechanism is random, only the missing observations will be excluded.

- If some dependent variable data are missing and the underlying mechanism is non-random, we will estimate group effects according to methods proposed by $\mathrm{Wu}$ and Bailey ${ }^{64}$ and Milliken and Johnson. ${ }^{65}$

Violations of the missing-at-random assumption will be investigated by following established precedents in paediatric oncology studies.

\section{Data management}

All patients enrolled in the study are given a unique identifier. A Microsoft Access database has been developed to record and link all the sociodemographic and clinical data for a study participant with information from their radiology assessments. Data protection regulations at 
each centre will be complied with. Data will be submitted centrally via a secure National Health Service email address with all patient identifiers removed. At each hospital site, local clinicians and physiotherapists will complete the relevant forms at each time point, with forms anonymised locally prior to being returned to the central trial unit. Images of MRI scans are to be anonymised locally and placed onto CDs, which are to be sent to the central trial unit. DXA scan images and reports are to be anonymised locally and sent to the central trial unit.

At present, data are not published in a data repository.

The full protocol is available in online supplementary file 2. Sample consent forms and patient information sheets are available as online supplementary file 3 .

\section{Protocol amendments}

All substantial protocol amendments will be agreed with the protocol contributors and require research ethics committee approval. Modifications will be communicated to the relevant parties via the website, newsletters and email.

\section{Ethics and dissemination}

NHS code of confidentiality and data protection will be adhered to. All data acquisition, storage and transmission will comply with the Data Protection Act 1998. The local clinical team will identify and provide age-appropriate patient information sheets to potential participants. Written patient consent or assent will be obtained by the local clinical team, with parental consent obtained for patients under 16 years of age. The protocol document and data collection tools are available online (http:// childhealth.leeds.ac.uk/bones.html). All substantial protocol contributors will be granted authorship of the final study report. There are no plans to use professional medical writers.

Collective results of the study will be published on the website, in peer-reviewed journals and presented at relevant conferences and via social media.

\section{Author affiliations}

${ }^{1}$ Leeds Institute of Cancer and Pathology, University of Leeds, Leeds, UK ${ }^{2}$ Department of Paediatric Haematology, Leeds Children's Hospital, Leeds, UK ${ }^{3}$ Department of Epidemiology, University of Leeds, Leeds, UK ${ }^{4}$ Department of Radiology, Leeds Teaching Hospital NHS Trust, Leeds, UK ${ }^{5}$ Department of Physiotherapy, Leeds Children's Hospital, Leeds, UK

${ }^{6}$ Department of Paediatric Haematology, Birmingham Women's and Children's NHS Foundation Trust, Birmingham, UK

Contributors All authors contributed to develop the protocol, helped to write and review the manuscript and made the decision to submit the manuscript for publication.

Funding This work was supported by a Candlelighters fellowship and Leeds Hospital Charitable Foundation. Fund no: 5T49/Approval No: 151

Disclaimer Sponsors and funders have no role in study design, collection, management, analysis and interpretation of data, writing of the report and decision to submit for publication

\section{Competing interests None declared.}

Patient consent for publication Not required.

Provenance and peer review Not commissioned; externally peer reviewed.
Open access This is an open access article distributed in accordance with the Creative Commons Attribution Non Commercial (CC BY-NC 4.0) license, which permits others to distribute, remix, adapt, build upon this work non-commercially, and license their derivative works on different terms, provided the original work is properly cited, appropriate credit is given, any changes made indicated, and the use is non-commercial. See: http://creativecommons.org/licenses/by-nc/4.0/.

\section{REFERENCES}

1. NCIN. National Registry of Childhood Tumours. Progress report, 2012. 2012

2. Amin N, Kinsey S, Feltbower R, et al. Prevalence, management, and long-term outcomes of osteonecrosis in young people with acute lymphoblastic leukaemia. Endocrine Abstracts 2015;39.

3. Barrack RL. Symptomatic multifocal osteonecrosis: a multicenter study. Clinical orthopaedics and related research 1999;369:312-26.

4. Kardos G, et al. Avascular necrosis of bone in children with acute lymphoblastic leukemia. Med Pediatr Oncol 1995;25:286.

5. Hanada T, Horigome Y, Inudoh M, et al. Osteonecrosis of vertebrae in a child with acute lymphocytic leukaemia during L-asparaginase therapy. Eur J Pediatr 1989;149:162-3.

6. Liu C, Janke LJ, Kawedia JD, et al. Asparaginase Potentiates Glucocorticoid-Induced Osteonecrosis in a Mouse Model. PLoS One 2016;11:e0151433.

7. Yang L, Panetta JC, Cai X, et al. Asparaginase may influence dexamethasone pharmacokinetics in acute lymphoblastic leukemia. $J$ Clin Oncol 2008;26:1932-9.

8. Girard P, Auquier P, Barlogis V, et al. Symptomatic osteonecrosis in childhood leukemia survivors: prevalence, risk factors and impact on quality of life in adulthood. Haematologica 2013;98:1089-97.

9. Kadan-Lottick NS, Dinu I, Wasilewski-Masker K, et al. Osteonecrosis in adult survivors of childhood cancer: a report from the childhood cancer survivor study. J Clin Oncol 2008;26:3038-45.

10. Strauss AJ, Su JT, Dalton VM, et al. Bony morbidity in children treated for acute lymphoblastic leukemia. $J$ Clin Oncol 2001;19:3066-72.

11. Mitchell CD, et al. Benefit of dexamethasone compared with prednisolone for childhood acute lymphoblastic leukaemia: results of the UK Medical Research Council ALL97 randomized trial. British journal of haematology 2005;129:734-45.

12. Kerachian MA, Séguin C, Harvey EJ. Glucocorticoids in osteonecrosis of the femoral head: a new understanding of the mechanisms of action. J Steroid Biochem Mol Biol 2009;114:121-8.

13. JONES JP. Fat Embolism, Intravascular Coagulation, and Osteonecrosis. Clin Orthop Relat Res 1993;292:294-308.

14. te Winkel ML, Appel IM, Pieters R, et al. Impaired dexamethasonerelated increase of anticoagulants is associated with development of osteonecrosis in childhood acute lymphoblastic leukaemia. Bone 2009;45:S106

15. Kaste SC, Pei D, Cheng C, et al. Utility of early screening magnetic resonance imaging for extensive hip osteonecrosis in pediatric patients treated with glucocorticoids. J Clin Oncol 2015;33:610-5.

16. Krull K, Kunstreich M, Klasen-Sansone J, et al. Osteonecrosis develops independently from radiological leukemic infiltration of bone in adolescents with acute lymphoblastic leukemia - first findings of the OPAL trial. Leuk Lymphoma 2017:58:2363-9.

17. Aricò M, Boccalatte MF, Silvestri D, et al. Osteonecrosis: An emerging complication of intensive chemotherapy for childhood acute lymphoblastic leukemia. Haematologica 2003;88:747-53.

18. Bürger B, Beier R, Zimmermann M, et al. Osteonecrosis: a treatment related toxicity in childhood acute lymphoblastic leukemia (ALL)-experiences from trial ALL-BFM 95. Pediatr Blood Cancer 2005;44:220-5.

19. Chen $\mathrm{S}-\mathrm{H}$, Chang $\mathrm{T}-\mathrm{Y}$, Jaing $\mathrm{T}-\mathrm{H}$, et al. Incidence, risk factors, and treatment outcome of symptomatic osteonecrosis in Taiwanese children with acute lymphoblastic leukemia: a retrospective cohort study of 245 patients in a single institution. Int $\mathrm{J} \mathrm{Hematol}$ 2015;102:41-7

20. Heneghan MB, Rheingold SR, Li Y, et al. Treatment of Osteonecrosis in Children and Adolescents With Acute Lymphoblastic Leukemia. Clinical Lymphoma Myeloma and Leukemia 2016;16:223-9.

21. Sakamoto K, Imamura T, Kihira K, et al. Low Incidence of Osteonecrosis in Childhood Acute Lymphoblastic Leukemia Treated With ALL-97 and ALL-02 Study of Japan Association of Childhood Leukemia Study Group. J Clin Oncol 2018;36:900-7.

22. Patel B, Richards SM, Rowe JM, et al. High incidence of avascular necrosis in adolescents with acute lymphoblastic leukaemia: a UKALL XII analysis. Leukemia 2008;22:308-12. 
23. Hough R, Rowntree C, Goulden N, et al. Efficacy and toxicity of a paediatric protocol in teenagers and young adults with Philadelphia chromosome negative acute lymphoblastic leukaemia: results from UKALL 2003. Br J Haematol 2016:172:439-51.

24. Kawedia JD, Kaste SC, Pei D, et al. Pharmacokinetic, pharmacodynamic, and pharmacogenetic determinants of osteonecrosis in children with acute lymphoblastic leukemia. Blood 2011;117:2340-7.

25. Relling MV, Yang W, Das S, et al. Pharmacogenetic risk factors for osteonecrosis of the hip among children with leukemia. J Clin Oncol 2004;22:3930-6.

26. Toft $\mathrm{N}$, Birgens $\mathrm{H}$, Abrahamsson J, et al. Toxicity profile and treatment delays in NOPHO ALL2008-comparing adults and children with Philadelphia chromosome-negative acute lymphoblastic leukemia. Eur J Haematol 2016;96.

27. Padhye B, Dalla-Pozza L, Little D, et al. Incidence and outcome of osteonecrosis in children and adolescents after intensive therapy for acute lymphoblastic leukemia (ALL). Cancer Med 2016;5.

28. Kunstreich M, Kummer S, Laws HJ, et al. Osteonecrosis in children with acute lymphoblastic leukemia. Haematologica 2016;101.

29. Mattano LA, Devidas M, Nachman JB, et al. Effect of alternateweek versus continuous dexamethasone scheduling on the risk of osteonecrosis in paediatric patients with acute lymphoblastic leukaemia: results from the CCG-1961 randomised cohort trial. Lancet Oncol 2012;13:906-15.

30. French D, Hamilton LH, Mattano LA, et al. A PAI-1 (SERPINE1) polymorphism predicts osteonecrosis in children with acute lymphoblastic leukemia: a report from the Children's Oncology Group. Blood 2008;111:4496-9.

31. Mitchell CD, Richards SM, Kinsey SE, et al. Benefit of dexamethasone compared with prednisolone for childhood acute lymphoblastic leukaemia: results of the UK Medical Research Council ALL97 randomized trial. Br J Haematol 2005;129:734-45.

32. te Winkel ML, Pieters R, Hop WC, et al. Prospective study on incidence, risk factors, and long-term outcome of osteonecrosis in pediatric acute lymphoblastic leukemia. J Clin Oncol 2011;29.

33. Mattano LA, Sather HN, Trigg ME, et al. Osteonecrosis as a complication of treating acute lymphoblastic leukemia in children: a report from the Children's Cancer Group. J Clin Oncol 2000;18:3262-72.

34. Niinimäki RA, Harila-Saari AH, Jartti AE, et al. High body mass index increases the risk for osteonecrosis in children with acute lymphoblastic leukemia. J Clin Oncol 2007;25:1498-504.

35. Karol SE, Yang W, Van Driest SL, et al. Genetics of glucocorticoidassociated osteonecrosis in children with acute lymphoblastic leukemia. Blood 2015;126.

36. Mogensen SS, Harila-Saari A, Mäkitie O, et al. Comparing osteonecrosis clinical phenotype, timing, and risk factors in children and young adults treated for acute lymphoblastic leukemia. Pediatr Blood Cancer 2018;65:e27300.

37. Salem KH, Brockert AK, Mertens R, et al. Avascular necrosis after chemotherapy for haematological malignancy in childhood. Bone Joint J 2013;95-B:1708-13.

38. Elmantaser M, Stewart G, Young D, et al. Skeletal morbidity in children receiving chemotherapy for acute lymphoblastic leukaemia. Arch Dis Child 2010;95:S109-S110.

39. Relling MV, Boyett JM, Blanco JG, et al. Granulocyte colonystimulating factor and the risk of secondary myeloid malignancy after etoposide treatment. Blood 2003;101:3862-7.

40. Ribeiro RC, Fletcher BD, Kennedy W, et al. Magnetic resonance imaging detection of avascular necrosis of the bone in children receiving intensive prednisone therapy for acute lymphoblastic leukemia or non-Hodgkin lymphoma. Leukemia 2001;15:891-7.

41. Badhiwala JH, Nayiager T, Athale UH. The development of thromboembolism may increase the risk of osteonecrosis in children with acute lymphoblastic leukemia. Pediatr Blood Cancer 2015;62.

42. Mogensen SS, Schmiegelow K, Grell K, et al. Hyperlipidemia is a risk factor for osteonecrosis in children and young adults with acute lymphoblastic leukemia. Haematologica 2017;102:e175-e178.
43. Karol SE, Mattano LA, Yang W, et al. Genetic risk factors for the development of osteonecrosis in children under age 10 treated for acute lymphoblastic leukemia. Blood 2016;127.

44. Ramsey LB, Pounds S, Cheng C, et al. Genetics of pleiotropic effects of dexamethasone. Pharmacogenet Genomics 2017;27:294-302.

45. Mont MA, Marulanda GA, Jones LC, et al. Systematic analysis of classification systems for osteonecrosis of the femoral head. J Bone Joint Surg Am 2006;88(suppl 3):16-26.

46. Arlet J, Ficat R. Forage-biopsie de la tête fémorale dans l'ostéonécrose primitive. Observations histopathologiques portant sur huit forages. Rev Rhum Mal Osteoartic 1964;31:257.

47. Steinberg ME, Hayken GD, Steinberg DR. A quantitative system for staging avascular necrosis. J Bone Joint Surg Br 1995;77:34-41.

48. Ono K. Diagnostic criteria, staging system and roentgenographic classification of avascular necrosis of the femoral head (steroid induced, alcohol associated or idiopathic nature), in Annual Report of Japanese Investigation Committee for Intractable Disease, O. K, Editor. Tokyo: Ministry of Health and Welfare, 1987:331-6.

49. Gardeniers J. ARCO international classification of osteonecrosis. ARCO News 1993;5:79-82.

50. Niinimäki T, Niinimäki J, Halonen J, et al. The classification of osteonecrosis in patients with cancer: validation of a new radiological classification system. Clin Radiol 2015;70:1439-44.

51. Niinimäki T, Harila-Saari A, Niinimäki R. The diagnosis and classification of osteonecrosis in patients with childhood leukemia. Pediatr Blood Cancer 2015;62:198-203.

52. Murphey MD, Foreman KL, Klassen-Fischer MK, et al. From the radiologic pathology archives imaging of osteonecrosis: radiologicpathologic correlation. Radiographics 2014;34:1003-28.

53. Vande Berg BE, Malghem JJ, Labaisse MA, et al. MR imaging of avascular necrosis and transient marrow edema of the femoral head. Radiographics 1993;13:501-20.

54. lida S, Harada Y, Shimizu K, et al. Correlation between bone marrow edema and collapse of the femoral head in steroid-induced osteonecrosis. AJR Am J Roentgenol 2000;174:735-43.

55. Singh G, Athreya BH, Fries JF, et al. Measurement of health status in children with juvenile rheumatoid arthritis. Arthritis Rheum 1994;37:1761-9.

56. Flatø $\mathrm{B}$, Aasland $\mathrm{A}$, Vandvik $\mathrm{IH}$, et al. Outcome and predictive factors in children with chronic idiopathic musculoskeletal pain. Clin Exp Rheumatol 1997;15:569-77.

57. Huber AM, Hicks JE, Lachenbruch PA, et al. Validation of the Childhood Health Assessment Questionnaire in the juvenile idiopathic myopathies. Juvenile Dermatomyositis Disease Activity Collaborative Study Group. J Rheumatol 2001;28:1106-11.

58. Meiorin S, Pistorio A, Ravelli A, et al. Validation of the Childhood Health Assessment Questionnaire in active juvenile systemic lupus erythematosus. Arthritis Rheum 2008;59:1112-9.

59. von Elm E, Altman DG, Egger M, et al. The Strengthening the Reporting of Observational Studies in Epidemiology (STROBE) statement: guidelines for reporting observational studies. PLoS Med 2007;4:e296.

60. Crabtree NJ, Shaw NJ, Bishop NJ, et al. Amalgamated Reference Data for Size-Adjusted Bone Densitometry Measurements in 3598 Children and Young Adults-the ALPHABET Study. J Bone Miner Res 2017:32:172-80.

61. Genant HK, Jergas M. Assessment of prevalent and incident vertebral fractures in osteoporosis research. Osteoporos Int 2003;14:43-55

62. Textor J, van der Zander B, Gilthorpe MS, et al. Robust causal inference using directed acyclic graphs: the R package 'dagitty'. Int $J$ Epidemiol 2016;45:1887-94

63. Ministry of housing. The English Index of Multiple Deprivation 2015, c.a.l.g. 2015

64. Wu MC, Bailey KR. Estimation and comparison of changes in the presence of informative right censoring: conditional linear model. Biometrics 1989;45:939-55.

65. Milliken GA, Johnson DE. Analysis of messy data volume 1: designed experiments: CRC Press, 2009:1. 